\title{
Semicircle law for a matrix ensemble with dependent entries
}

\author{
Winfried Hochstättler, Werner Kirsch \\ Fakultät für Mathematik und Informatik \\ FernUniversität in Hagen, Germany \\ Simone Warzel \\ Zentrum Mathematik \\ Technische Universität München, Germany
}

\begin{abstract}
We study ensembles of random symmetric matrices whose entries exhibit certain correlations. Examples are distributions of Curie-Weiss-type. We provide a criterion on the correlations ensuring the validity of Wigner's semicircle law for the eigenvalue distribution measure. In case of CurieWeiss distributions this criterion applies above the critical temperature (i. e. $\beta<1$ ). We also investigate the largest eigenvalue of certain ensembles of Curie-Weiss type and find a transition in its behavior at the critical temperature.
\end{abstract}

\section{Introduction}

In this article we consider random matrices $X_{N}$ of the form

$$
X_{N}=\left(\begin{array}{cccc}
X_{N}(1,1) & X_{N}(1,2) & \ldots & X_{N}(1, N) \\
X_{N}(2,1) & X_{N}(2,2) & \ldots & X_{N}(2, N) \\
\vdots & \vdots & & \vdots \\
X_{N}(N, 1) & X_{N}(N, 2) & \ldots & X_{N}(N, N)
\end{array}\right)
$$

The entries $X_{N}(i, j)$ are real valued random variables varying with $N$. We will always assume that the matrix $X_{N}$ is symmetric, such that 
$X_{N}(i, j)=X_{N}(j, i)$ for all $i, j$. Furthermore we suppose that all moments of the $X_{N}(i, j)$ exist and that $\mathbb{E}\left(X_{N}(i, j)\right)=0$ and $\mathbb{E}\left(X_{N}(i, j)^{2}\right)=1$.

It is convenient to work with the normalized version $A_{N}$ of $X_{N}$, namely with

$$
A_{N}=\frac{1}{\sqrt{N}} X_{N}
$$

As $A_{N}$ is symmetric it has exactly $N$ real eigenvalues (counting multiplicity). We denote them by

$$
\lambda_{1} \leq \lambda_{2} \leq \ldots \leq \lambda_{N}
$$

and define the (empirical) eigenvalue distribution measure by

$$
\sigma_{N}=\frac{1}{N} \sum_{j=1}^{N} \delta_{\lambda_{j}}
$$

and its expected value $\bar{\sigma}_{N}$, the density of states measure by

$$
\bar{\sigma}_{N}=\mathbb{E}\left(\frac{1}{N} \sum_{j=1}^{N} \delta_{\lambda_{j}}\right)
$$

If the random variables $X_{N}(i, j)$ are independent and identically distributed (i.i.d.) (except for the symmetry condition $X_{N}(i, j)=X_{N}(j, i)$ ) then it is well known that the measures $\bar{\sigma}_{N}$ and $\sigma_{N}$ converge weakly to the semicircle distribution $\sigma_{s c}$ (almost surely in the case of $\sigma_{N}$ ). The semicircle distribution is concentrated on the interval $[-2,2]$ and has a density given by $\sigma_{s c}(x)=\frac{1}{2 \pi} \sqrt{4-x^{2}}$ for $x \in[-2,2]$. This important result is due to Eugen Wigner [23] and was proved by Arnold [3] in greater generality, see also for example [17], [18] or [2].

Recently, there was a number of papers considering random matrices with some kind of dependence structure among their entries, see for example [7], [13], [12] and [20]. In particular the papers [6], [10] and [11] consider symmetric random matrices whose entries $X_{N}(i, j)$ and $X_{N}(k, \ell)$ are independent if they belong to different diagonals, i.e. if $|i-j| \neq|k-\ell|$, but may be dependent within the diagonals. It was in particular the work [11] which motivated the current paper. Among other models Friesen and Löwe [11] consider matrices with independent diagonals and (independent copies of) Curie-Weiss distributed random variables on the diagonals. (For a definition of the Curie-Weiss model see below).

The main example for the results in our paper is a symmetric random matrix whose entries $X_{N}(i, j)$ are Curie-Weiss distributed for all $i, j$ (with $i \leq j$ ). The models considered in this paper also include the Curie-Weiss 
model on diagonals investigated by Friesen and Löwe. For the reader's convenience we define our Curie-Weiss ensemble here, but we'll work with abstract assumptions in the following two chapters.

In statistical physics the Curie-Weiss model serves as the easiest nontrivial model of magnetism. There are $M$ sites with random variables $X_{i}$ attached to the sites $i$ taking values +1 ("spin up") or -1 ("spin down"). Each spin $X_{i}$ interacts with all the other spins prefering to be aligned with the average spin $\frac{1}{M} \sum_{j \neq i} X_{j}$. More precisely:

Definition 1 Random variables $\left\{X_{j}\right\}_{j=1, \ldots, M}$ with values in $\{-1,+1\}$ are distributed according to a Curie-Weiss law $\mathbb{P}_{\beta, M}$ with parameters $\beta \geq 0$ (called the inverse temperature) and $M \in \mathbb{N}$ (called the number of spins) if

$$
\mathbb{P}_{\beta, M}\left(X_{1}=\xi_{1}, X_{2}=\xi_{2}, \ldots, X_{M}=\xi_{M}\right)=Z_{\beta, M}^{-1} \frac{1}{2^{M}} e^{\frac{\beta}{2 M}\left(\sum \xi_{j}\right)^{2}}
$$

where $\xi_{i} \in\{-1,+1\}$ and $Z_{\beta, M}$ is a normalization constant.

For $\beta<1$ Curie-Weiss distributed random variables are only weakly correlated, while for $\beta>1$ they are strongly correlated. This is expressed for example by the fact that a law of large numbers holds for $\beta<1$, but is wrong for $\beta>1$. This sudden change of behavior is called a "phase transition" in physics. In theoretical physics jargon the quantity $T=\frac{1}{\beta}$ is called the temperature and $T=1$ is called the critical temperature. More information about the Curie-Weiss model and its physical meaning can be found in [22] and [8].

Our Curie-Weiss matrix model, which we dub the full Curie-Weiss ensemble, is defined through $M=N^{2}$ random variables $\left\{Y_{N}(i, j)\right\}_{1 \leq i, j \leq N}$ which are $\mathbb{P}_{\beta, M}$-distributed. To form a symmetric matrix we set $X_{N}(i, j)=$ $Y_{N}(i, j)$ for $i \leq j$ and $X_{N}(i, j)=Y_{N}(j, i)$ for $i>j$ and define

$$
A_{N}=\frac{1}{\sqrt{N}} X_{N}
$$

By the diagonal Curie-Weiss ensemble we mean a symmetric random matrix with the random variables on the $k^{\text {th }}$ diagonal $\{i, i+k\}$ being $\mathbb{P}_{\beta, N^{-}}$ distributed $(0 \leq k \leq N-1$ and $1 \leq i \leq N-k)$ and with entries on different diagonals being independent. This model was considered in [11]. For $\beta<1$ we will prove the semicircle law for these two ensembles.

In the following section we formulate our general abstract assumptions and state the first theorem of this paper which establishes the semicircle law for our models. The proof follows in Section 3

In Section 4 we discuss our main example, the full Curie-Weiss model, in fact we will study various random matrix ensembles associated to CurieWeiss-like models. In this section we also discuss exchangeable random variables and their connection with the Curie-Weiss model. 
In Section 5 we investigate the largest eigenvalue (and thus the matrix norm) of Curie-Weiss-type matrix ensembles both below and above the critical value $\beta=1$.

Acknowlegment It is a pleasure to thank Matthias Löwe, Münster, and Wolfgang Spitzer, Hagen, for valuable discussion. Two of us (WK and SW) would like to thank the Institute for Advanced Study in Princeton, USA, where part of this work was done, for support and hospitality.

\section{The semicircle law}

Definition 2 Suppose $\left\{I_{N}\right\}_{N \in \mathbb{N}}$ is a sequence of finite index sets $I_{N}$. A family $\left\{X_{N}(\rho)\right\}_{\rho \in I_{N}, N}$ of random variables indexed by $N \in \mathbb{N}$ and (for given $N$ ) by the set $I_{N}$ is called an $\left\{I_{N}\right\}$-scheme of random variables. If the sequence $\left\{I_{N}\right\}$ is clear from the context we simply speak of a scheme.

To define an ensemble of symmetric random matrices we start with a 'quadratic' scheme of random variables $\left\{Y_{N}(i, j)\right\}_{(i, j) \in I_{N}}$ with $I_{N}=$ $\{(i, j) \mid 1 \leq i, j \leq N\}$ and define the matrix entries $X_{N}(i, j)$ by $X_{N}(i, j)=$ $Y_{N}(i, j)$ for $i \leq j$ and $X_{N}(i, j)=Y_{N}(j, i)$ for $i>j$.

Remark 3 To define the symmetric matrix $X_{N}$ it would be enough to start with a 'triangular' scheme of random variables, i.e. one with $I_{N}=\{(i, j) \mid 1 \leq i \leq j \leq N\}$, thus with $M=\frac{1}{2} N(N+1)$ random variables instead of $M=N^{2}$ variables. To reduce notational inconvenience we decided to use the quadratic schemes. In a slight abuse of language we will no longer distiguish in notation between the random variables $Y_{N}(i, j)$ and their symmetrized version $X_{N}(i, j)$. We will always assume that the random matrices we are dealing with are symmetric.

In this paper we consider schemes $\left\{X_{N}(i, j)\right\}_{(i, j) \in I_{N}}$ of random variables with $N=1,2, \ldots$ and $I_{N}=\{(i, j) \mid 1 \leq i, j \leq N\}$ with the following property:

Definition 4 A scheme $\left\{X_{N}(i, j)\right\}_{(i, j) \in I_{N}}$ is called approximately uncorrelated, if

$$
\begin{aligned}
&\left|\mathbb{E}\left(\prod_{\nu=1}^{\ell} X_{N}\left(i_{\nu}, j_{\nu}\right) \prod_{\rho=1}^{m} X_{N}\left(u_{\rho}, v_{\rho}\right)\right)\right| \leq \frac{C_{\ell, m}}{N^{\ell / 2}} \\
&\left|\mathbb{E}\left(\prod_{\nu=1}^{\ell} X_{N}\left(i_{\nu}, j_{\nu}\right)^{2}\right)-1\right| \rightarrow 0
\end{aligned}
$$

for all sequences $\left(i_{1}, j_{1}\right),\left(i_{2}, j_{2}\right), \ldots,\left(i_{\ell}, j_{\ell}\right)$ which are pairwise disjoint and disjoint to the sequence $\left(u_{1}, v_{1}\right), \ldots,\left(u_{m}, v_{m}\right)$ with $N$-independent constants $C_{\ell, m}$. 
Note that for any approximately correlated scheme the mean asymptotically vanishes, $\left|\mathbb{E}\left(X_{N}((i, j))\right)\right| \leq C_{1,0} N^{-1 / 2}$ by (4), and the variance is asymptotically one, $\mathbb{E}\left(X_{N}(i, j)^{2}\right) \rightarrow 1$ by (5). Moreover, by (4) we also have $\sup _{N, i, j} \mathbb{E}\left(X_{N}(i, j)^{2 k}\right)<\infty$ for all $k$.

The main examples we have in mind are schemes of Curie-Weiss- distributed random variables (full or diagonal) with inverse temperature $\beta \leq 1$ (for details see Section 4).

Theorem 5 If $\left\{X_{N}(i, j)\right\}_{1 \leq i \leq j \leq N}$ is an approximately uncorrelated scheme of random variables then the eigenvalue distribution measures $\sigma_{N}$ of the corresponding symmetric matrices $A_{N}$ (as in (2)) converge weakly in probability to the semicircle law $\sigma_{s c}$, i.e. for all bounded continuous functions $f$ on $\mathbb{R}$ and all $\varepsilon>0$ we have

$$
\mathbb{P}\left(\left|\int f(x) d \sigma_{N}(x)-\int f(x) d \sigma_{s c}(x)\right|>\varepsilon\right) \rightarrow 0 .
$$

In particular, we prove the weak convergence of the density of states measure $\bar{\sigma}_{N}$ to the semicircle law $\sigma_{s c}$. In Section 4 we discuss various examples of approximately uncorrelated schemes.

\section{Proof of the semicircle law}

The proof is a refinement of the classical moment method (see for example [2]). We will sketch the proof emphasizing only the new ingredients. As in [2], Theorem 5 follows from the following two propositions.

Proposition 6 For all $k \in \mathbb{N}$ :

$$
\frac{1}{N} \mathbb{E}\left(\operatorname{tr} A^{k}\right) \rightarrow\left\{\begin{array}{cc}
C_{k / 2} & \text { for } k \text { even } \\
0 & \text { for } k \text { odd }
\end{array}\right.
$$

where $C_{k}=\frac{1}{k+1}\left(\begin{array}{c}2 k \\ k\end{array}\right)$ denote the Catalan numbers.

The right hand side of (6) gives the moments of the semicircle distribution $\sigma_{s c}$. In fact, this proposition implies the weak convergence of the density of states measures $\bar{\sigma}_{N}$ to $\sigma_{s c}$.

Proposition 7 For all $k \in \mathbb{N}$ :

$$
\frac{1}{N^{2}} \mathbb{E}\left[\left(\operatorname{tr} A^{k}\right)^{2}\right] \rightarrow\left\{\begin{array}{cc}
C_{k / 2}^{2} & \text { for } k \text { even } \\
0 & \text { for } k \text { odd }
\end{array} .\right.
$$


Observe that Proposition 6 and Proposition 7 together imply that

$$
\mathbb{E}\left[\left(\frac{1}{N} \operatorname{tr} A^{k}\right)^{2}\right]-\mathbb{E}\left[\left(\frac{1}{N} \operatorname{tr} A^{k}\right)\right]^{2} \rightarrow 0
$$

which allows us to conclude weak convergence in probability from weak convergence in the average (see [2]).

For a proof of the above propositions, which can be found in the subsequent subsections, we write

$$
\begin{aligned}
\frac{1}{N} \operatorname{tr} A^{k} & =\frac{1}{N^{1+k / 2}} \sum_{i_{1}, i_{2}, \ldots, i_{k}=1}^{N} X_{N}\left(i_{1}, i_{2}\right) X_{N}\left(i_{2}, i_{3}\right) \ldots X_{N}\left(i_{k}, i_{1}\right) \\
& =\frac{1}{N^{1+k / 2}} \sum_{i_{1}, i_{2}, \ldots, i_{k}=1}^{N} X_{N}(\underline{i})
\end{aligned}
$$

where we used the short hand notation

$$
X_{N}(\underline{i})=X_{N}\left(i_{1}, i_{2}\right) X_{N}\left(i_{2}, i_{3}\right) \ldots X_{N}\left(i_{k}, i_{1}\right)
$$

for $\underline{i}=\left(i_{1}, i_{2}, \ldots, i_{k}\right)$. The associated $k+1$-tupel $\left(i_{1}, i_{2}, \ldots, i_{k}, i_{1}\right)$ constitutes a Eulerian circuit through the graph $\mathcal{G}_{\underline{i}}$ (undirected, not necessary simple) with vertex set $V_{i}=\left\{i_{1}, i_{2}, \ldots, i_{k}\right\}$ and an edge between the vertices $v$ and $w$ whenever $\{v, w\}=\left\{i_{j}, i_{j+1}\right\}$ for some $j=1, \ldots k$ with the understanding that $i_{k+1}=i_{1}$, a convention we keep for the rest of this paper. More precisely, the number of edges $\nu(v, w)$ linking the vertex $v$ and the vertex $w$ is given by

$$
\nu(v, w)=\#\left\{m \mid\{v, w\}=\left\{i_{m}, i_{m+1}\right\}\right\} .
$$

Let us call edges $e_{1} \neq e_{2}$ parallel if they link the same vertices. An edge which does not have a parallel edge is called simple. So, if $e$ links $v$ and $w$, then $e$ is a simple edge iff $\nu(v, w)=1$. The graph $\mathcal{G}_{\underline{i}}$ may contain loops, i.e. edges connecting a vertex $v$ with itself. By a proper edge we mean an edge which is not a loop. We set $\rho(\underline{i})=\#\left\{i_{1}, i_{2}, \ldots, i_{k}\right\}$ the cardinality of the vertex set $V_{\underline{i}}$, i.e., the number of (distinct) vertices the Eulerian circuit visits. We also denote by $\sigma(\underline{i})$ the number of simple edges in the Eulerian circuit $\left(i_{1}, i_{2}, \ldots, i_{k}, i_{1}\right)$. With this notation we can write (9) as

$$
\begin{aligned}
\frac{1}{N} \operatorname{tr} A^{k} & =\frac{1}{N^{1+k / 2}} \sum_{r=1}^{k} \sum_{\underline{i}: \rho(\underline{i})=r} X_{N}(\underline{i}) \\
& =\frac{1}{N^{1+k / 2}} \sum_{r=1}^{k} \sum_{s=0}^{k} \sum_{\substack{\rho(\underline{i})=r \\
\sigma(\underline{i})=s}} X_{N}(\underline{i}) .
\end{aligned}
$$


The sum extends over all Eulerian circuits with $k$ edges and vertex set $V_{\underline{i}} \subset\{1,2, \ldots, N\}$. To simplify future references we set

$$
S_{r, s}=\sum_{\substack{\rho(\underline{i})=r \\ \sigma(\underline{i})=s}}\left|\mathbb{E}\left[X_{N}(\underline{i})\right]\right|
$$

and

$$
S_{r}=\sum_{\rho(\underline{i})=r}\left|\mathbb{E}\left[X_{N}(\underline{i})\right]\right|
$$

Obviously $\rho(\underline{i})$ and $\sigma(\underline{i})$ are integers with $1 \leq \rho(\underline{i}) \leq k$ and $0 \leq \sigma(\underline{i}) \leq k$. For $\rho(\underline{i})=r<N$ there are $\left(\begin{array}{c}\bar{N} \\ r\end{array}\right) \leq N^{r}$ choices for the vertex set $V_{\underline{i}}$. Moreover,

$$
\#\{\underline{i} \mid \rho(\underline{i})=r\} \leq \eta_{k} N^{r}
$$

where $\eta_{k}$ is the number of equivalence classes of Eulerian circuits of length $k$. We call two Eulerian circuits $\left(i_{1}, i_{2}, \ldots, i_{k}, i_{1}\right)$ and $\left(j_{1}, j_{2}, \ldots, j_{k}, j_{1}\right)$ with corresponding vertex sets $V_{\underline{i}}$ and $V_{j}$ equivalent if there is a bijection $\varphi$ : $V_{\underline{i}} \rightarrow V_{\underline{j}}$ such that $\varphi\left(i_{m}\right)=\bar{j}_{m}$ for all $m$.

\subsection{Proof of Proposition 6}

We investigate the expectation value of the sum (12).

Lemma 8 For all $k \in \mathbb{N}$ there is some $D_{k}<\infty$ such that for all $N$ :

$$
S_{r, s}=\sum_{\substack{\rho(\underline{i})=r \\ \sigma(\underline{i})=s}}\left|\mathbb{E}\left[X_{N}(\underline{i})\right]\right| \leq D_{k} N^{r-s / 2} .
$$

Proof. The assertion follows using (4) from the estimate $\left|\mathbb{E}\left[X_{N}(\underline{i})\right]\right| \leq$ $\widetilde{D}_{k} N^{-s / 2}$ together with (15).

Evidently, in case $r-s / 2<1+k / 2$ the term $\frac{1}{N^{k / 2+1}} S_{r, s}$ vanishes in the limit. This is in particular the case if $r<k / 2+1$. If $r>k / 2+1$ we use the following proposition which is one of the key ideas of our proof:

Proposition 9 Let $\mathcal{G}=(V, E)$ denote a Eulerian graph with $r=\# V$ and $k=\# E$, and let $t$ be a positive integer such that $r>\frac{k}{2}+t$ then $\mathcal{G}$ has at least $2 t+1$ simple proper edges.

We note the following Corollary to Proposition 9 
Corollary 10 For each $k$-tuple $\underline{i}$ we have

$$
\rho(\underline{i})-\sigma(\underline{i}) / 2 \leq k / 2+1 .
$$

Moreover $\rho(\underline{i})-\sigma(\underline{i}) / 2=k / 2+1$ iff $\rho(\underline{i})=k / 2+1$ and $\sigma(\underline{i})=0$.

Proof (Corollary 10). Set $r=\rho(\underline{i})$ and $s=\sigma(\underline{i})$.

If $r \leq k / 2+1$ the assertion is evident.

If $r>k / 2+1$ there is some $t \in \mathbb{N}$ such that

$$
\frac{k}{2}+t<r \leq \frac{k}{2}+t+1 .
$$

Proposition 9 hence implies

$$
r-\frac{s}{2} \leq r-t-\frac{1}{2} \leq \frac{k}{2}+\frac{1}{2}<\frac{k}{2}+1 .
$$

Postponing the proof of Proposition 9, we continue to prove Proposition 6 .

From Lemma 8 and Corollary 10 we learn that

$$
\frac{1}{N^{k / 2+1}} S_{r, s} \rightarrow 0
$$

unless both $r=k / 2+1$ and $s=0$.

Thus it remains to compute the number of $k$-tuples $\underline{i}$, with $\rho(\underline{i})=1+k / 2$ such that the corresponding graph is a 'doubled' planar tree. There are $C_{\frac{k}{2}}$ different rooted planar trees with $\frac{k}{2}$ (simple) edges, where $C_{\ell}=\frac{1}{\ell+1}\left(\begin{array}{c}2 \ell \\ \ell\end{array}\right)$ are the Catalan numbers (see e.g. [19], Exercise 6.19 e, p. 219-220). Each index $i_{\nu}$ is chosen from the set $\{1, \ldots, N\}$. As we have $1+k / 2$ different indices there are $\frac{N !}{(N-1-k / 2) !}$ such choices. From this one sees that

$$
\begin{aligned}
\lim _{N \rightarrow \infty} \frac{1}{N} \mathbb{E}\left(\operatorname{tr} A^{k}\right) & =\lim _{N \rightarrow \infty} \sum_{r=1}^{k} \frac{1}{N^{1+k / 2}} \sum_{\rho(\underline{i})=r} \mathbb{E}\left(X_{N}(\underline{i})\right) \\
& =\lim _{N \rightarrow \infty} \frac{1}{N^{1+k / 2}} \sum_{\rho(\underline{i})=1+k / 2, \sigma(\underline{i})=0} \mathbb{E}\left(X_{N}(\underline{i})\right) \\
& =\lim _{N \rightarrow \infty} \frac{1}{N^{1+k / 2}} \frac{N !}{(N-1-k / 2) !} C_{\frac{k}{2}}=C_{\frac{k}{2}} .
\end{aligned}
$$

This ends the proof of Lemma 6 modulo the proof of the Proposition 9 For future purpose, we note that the above proof also shows the slightly stronger assertion. 
Corollary 11 For all $k \in \mathbb{N}$ :

$$
\lim _{N \rightarrow \infty} \frac{1}{N^{1+k / 2}} \sum_{\underline{i}}\left|\mathbb{E}\left[X_{N}(\underline{i})\right]\right|=\left\{\begin{array}{cc}
C_{k / 2} & \text { for } k \text { even } \\
0 & \text { for } k \text { odd }
\end{array}\right.
$$

where the above sum (in (19)) extends over all Eulerian circuits of length $k$.

For a proof we note that the leading contribution in the sum (18) is nonnegative. The subleading terms were already shown to vanish.

Proof (Proposition 9). If the graph $\mathcal{G}=(V, E)$ contains loops, we delete all loops and call the new graph $(V, \tilde{E})$. This graph is still Eulerian and satisfies $\# V>\# \tilde{E} / 2+t$. Thus without loss of generality we may assume that $(V, E)$ contains no loops.

We proceed by induction on the number of edges with multiplicity greater than one. If there is no such edge, then the number of simple edges is $k$. Since $\mathcal{G}$ is Eulerian we have $k \geq r$ and $r>\frac{k}{2}+t$ implies $k>2 t$ and thus the assertion.

Hence, assume there exists an edge of multiplicity $m \geq 2$. If the graph that arises from the deletion of 2 copies of this edge is still connected, then the resulting graph is Eulerian and denoting its number of edges by $k^{\prime}$ we have

$$
r>\frac{k}{2}+t \geq \frac{k^{\prime}}{2}+t+1
$$

Thus, by inductive assumption, we find at least $2 t+3$ edges without parallels in the reduced graph and hence at least $2 t+2$ in $\mathcal{G}$.

We are left with the case that the removal of the edges disconnects the graph into two Eulerian graphs $\mathcal{G}_{1}=\left(V_{1}, E_{1}\right)$ and $\mathcal{G}_{2}=\left(V_{2}, E_{2}\right)$. We use the abbreviations $r_{i}:=\# V_{i}$ and $k_{i}:=\# E_{i}$. Then:

$$
r=r_{1}+r_{2}>\frac{k}{2}+t=\frac{k_{1}+1}{2}+\frac{k_{2}+1}{2}+t
$$

Hence we can partition $t$ into integers $t_{1}, t_{2}$ such that

$$
r_{1}>\frac{k_{1}}{2}+t_{1} \text { and } r_{2}>\frac{k_{2}}{2}+t_{2}
$$

If, say $t_{1} \leq 0$, then $t_{2} \geq t$ and the inductive assumption yields at least $2 t+1$ simple edges in $\mathcal{G}_{2}$ and hence in $\mathcal{G}$.

Otherwise we find at least $2 t_{i}+1$ simple edges in each of the $\mathcal{G}_{i}$ and thus in total $2 t+2>2 t+1$ such edges in $\mathcal{G}$. 


\subsection{Proof of Proposition 7}

We write the expectation value

$$
\frac{1}{N^{2}} \mathbb{E}\left[\left(\operatorname{tr} A^{k}\right)^{2}\right]=\frac{1}{N^{2+k}} \sum_{\underline{i}, \underline{j}} \mathbb{E}\left[X_{N}(\underline{i}) X_{N}(\underline{j})\right]
$$

where the sum extends over all pairs of Eulerian circuits $\left(i_{1}, \ldots, i_{k}, i_{1}\right)$ and $\left(j_{1}, \ldots, j_{k}, j_{1}\right)$ of length $k$ with vertex sets $V_{\underline{i}}$ and $V_{\underline{j}}$ in $\{1, \ldots, N\}$. We distinguish two cases.

In case $V_{\underline{i}} \cap V_{j} \neq \emptyset$ the union of the corresponding Eulerian graphs $\mathcal{G}_{\underline{i}} \cup \mathcal{G}_{j}$ is connected and each vertex has even degree. Therefore this union is itself a Eulerian graph with $2 k$ edges. The corresponding contribution to the sum (20) is then estimated by extending the summation to all Eulerian circuits $\underline{\ell}$ of length $2 k$ :

$$
\frac{1}{N^{2+k}} \sum_{\substack{\underline{i}, j \\ V_{\underline{i}} \cap V_{\underline{j}} \neq \emptyset}}\left|\mathbb{E}\left[X_{N}(\underline{i}) X_{N}(\underline{j})\right]\right| \leq \frac{1}{N^{2+k}} \sum_{\underline{\ell}=\left(\ell_{1}, \ldots, \ell_{2 k}\right)}\left|\mathbb{E}\left[X_{N}(\underline{\ell})\right]\right| \leq \frac{\widetilde{C}_{k}}{N} .
$$

The last estimate is due to Corollary 11

In case $V_{\underline{i}} \cap V_{\underline{j}}=\emptyset$ we use the following analogue of Lemma 8 .

Lemma 12 For all $k \in \mathbb{N}$ there is some $D_{k}<\infty$ such that for all $N$ :

$$
\sum_{\substack{V_{\underline{i}} \cap V_{j}=\emptyset \\ \rho(\underline{i})=r_{1}, \rho(j)=r_{2} \\ \sigma(\underline{i})=s_{1}, \sigma(\underline{j})=s_{2}}}\left|\mathbb{E}\left[X_{N}(\underline{i}) X_{N}(\underline{j})\right]\right| \leq D_{k} N^{r_{1}+r_{2}-s_{1} / 2-s_{2} / 2},
$$

where the sum extends over non-intersecting pairs of Eulerian circuits of length $k$.

The proof mirrors that of Lemma 8 ,

From Lemma 8 we know that $r_{1}-s_{1} / 2 \leq k / 2+1$ and likewise $r_{2}-$ $s_{2} / 2 \leq k / 2+1$. So the unique possibility that

$$
r_{1}+r_{2}-\frac{s_{1}+s_{2}}{2} \geq k+2
$$

giving rise to a non-vanishing term in the limit, is that $r_{1}=r_{2}=k / 2+$ 1 and $s_{1}=s_{2}=0$. Similarly as in the proof of Lemma 6 we conclude that in this case $\underline{i}$ and $\underline{j}$ constitute disjoint 'doubled' planar trees and $\mathbb{E}\left[X_{N}(\underline{i}) X_{N}(\underline{j})\right] \rightarrow 1$ by assumption (5). The proof of Lemma 7 is concluded using the same arguments relating the number of planar trees to the Catalan numbers. 


\section{The Curie-Weiss model and its relatives}

In this section we discuss the Curie-Weiss model and related ensembles in the framework of general exchangeable sequences. Let us first recall:

Definition 13 A finite sequence $X_{1}, X_{2}, \ldots, X_{M}$ of random variables is called exchangeable if for any permutation $\pi \in \mathcal{S}_{M}$ the joint distributions of $X_{1}, X_{2}, \ldots, X_{M}$ and of $X_{\pi(1)}, X_{\pi(2)}, \ldots, X_{\pi(M)}$ agree. An infinite sequence $\left\{X_{i}\right\}_{i \in I}$ is called exchangeable if any finite subsequence is.

It is a well known result by de Finetti ([9], for further developments see e.g. [1]) that any exchangeable sequence of $\{-1,1\}$-valued random variables is a mixture of independent random variables. To give this informal description a precise meaning we define:

Definition 14 For $t \in[-1,1]$ we denote by $P_{t}$ the probability measure $P_{t}=\frac{1}{2}(1+t) \delta_{1}+\frac{1}{2}(1-t) \delta_{-1}$ on $\{-1,1\}$, i.e. $P_{t}(1)=\frac{1}{2}(1+t)$ and $P_{t}(-1)=\frac{1}{2}(1-t)$. By $P_{t}^{M}=P_{t}^{\otimes_{M}}$ we mean the $M$-fold, by $P_{t}^{\infty}=P_{t}^{\otimes_{\mathbb{N}}}$ the infinite product of this measure.

Remark 15 The measures $P_{t}$ are parametrized in such a way that $E_{t}(X):=$ $\int x d P_{t}=t$. To simplify notation, we write $P_{t}^{M}\left(x_{1}, x_{2}, \ldots, x_{M}\right)$ instead of $P_{t}^{M}\left(\left\{\left(x_{1}, x_{2}, \ldots, x_{M}\right)\right\}\right)$.

We are now in a position to formulate de Finetti's theorem:

Theorem 16 (de Finetti) If $\left\{X_{i}\right\}_{i \in \mathbf{N}}$ is an exchangeable sequence of $\{-1,1\}$-valued random variables with distribution $\mathbb{P}\left(\right.$ on $\left.\{-1,1\}^{\mathbb{N}}\right)$ then there exists a probability measure $\mu$ on $[-1,1]$, such that for any measurable set $S \subset\{-1,1\}^{\mathbb{N}}$ :

$$
\mathbb{P}(S)=\int P_{t}^{\infty}(S) d \mu(t) .
$$

For this result it is essential that the index set $I=\mathbb{N}$ is infinite. In fact, the theorem does not hold for finite sequences in general (see e. g. [1]).

Definition 17 If $\mu$ is a probability measure on $[-1,1]$ then we call a measure

$$
\mathbb{P}(\cdot)=\int P_{t}^{M}(\cdot) d \mu(t)
$$

on $\{-1,1\}^{M}$ a measure of de Finetti type (with de Finetti measure $\mu$ ). We say that a finite sequence $\left\{X_{1}, X_{2}, \ldots, X_{M}\right\}$ of random variables is of de Finetti type if the joint distribution of the $\left\{X_{i}\right\}_{i=1}^{M}$ is of de Finetti type.

The following observation allows us to compute correlations of de Finetti type random variables: 
Proposition 18 If the sequence $\left\{X_{1}, X_{2}, \ldots, X_{M}\right\}$ of random variables is of de Finetti type with de Finetti measure $\mu$ then for distinct $i_{1}, \ldots, i_{K}$

$$
\mathbb{E}\left(X_{i_{1}} X_{i_{2}} \ldots X_{i_{K}}\right)=\int t^{K} d \mu(t) .
$$

Proof. By the definition of $P_{t}^{M}$ we have $E_{t}^{M}\left(X_{i_{1}} X_{i_{2}} \ldots X_{i_{K}}\right)=t^{K}$.

Corollary 19 Suppose $\mathbb{P}_{N}(\cdot)=\int P_{t}^{N^{2}}(\cdot) d \mu_{N}(t)$ is a sequence of measures of de Finetti type and $X_{N}$ is a random matrix ensemble corresponding to $\mathbb{P}_{N}$ via Definition 2 If for all $k \in \mathbb{N}$

$$
\int t^{K} d \mu_{N}(t) \leq \frac{C_{K}}{N^{K / 2}}
$$

for some constants $C_{K}$, then $X_{N}$ satisfies the semicircle law.

Proof. We prove that $\left\{X_{N}(i, j)\right\}$ is approximately uncorrelated in the sense of Definition (4) Since $X_{N}(i, j)^{2}=1$ property (5) is evident. Property (4) follows from (24) and Proposition 18.

Curie-Weiss distributed random variables turn out to be examples of de Finetti sequences. This fact is contained in a somewhat hidden way in physics textbooks (see for example [22, section 4-5]).

Theorem 20 Curie-Weiss $\left(\mathbb{P}_{\beta, M^{-}}\right)$distributed random variables $\left\{X_{1}, X_{2}, \ldots, X_{M}\right\}$ are of de Finetti type, more precisely

$$
\begin{aligned}
& \mathbb{P}_{\beta, M}\left(X_{1}=x_{1}, X_{2}=x_{2}, \ldots, X_{M}=x_{M}\right) \\
= & Z^{-1} \int_{-1}^{+1} P_{t}^{M}\left(x_{1}, x_{2}, \ldots, x_{M}\right) \frac{e^{-M F_{\beta}(t) / 2}}{1-t^{2}} d t
\end{aligned}
$$

where $F_{\beta}(t)=\frac{1}{\beta}\left(\frac{1}{2} \ln \frac{1+t}{1-t}\right)^{2}+\ln \left(1-t^{2}\right)$ and the normalization factor is given by $Z=\int \frac{e^{-M F_{\beta}(t) / 2}}{1-t^{2}} d t$. 
Proof. Using the observation $e^{\frac{z^{2}}{2}}=(2 \pi)^{-\frac{1}{2}} \int_{-\infty}^{+\infty} e^{-\frac{s^{2}}{2}+s z} d s$ (also known as Hubbard-Stratonovich transformation) we obtain

$$
\begin{aligned}
& \mathbb{P}_{\beta, M}\left(X_{1}=x_{1}, X_{2}=x_{2}, \ldots, X_{M}=x_{M}\right) \\
= & Z_{\beta, M}^{-1} \frac{1}{2^{M}} e^{\frac{\beta}{2 M}\left(\sum x_{j}\right)^{2}} \\
= & (2 \pi)^{-1 / 2} Z_{\beta, M}^{-1} \frac{1}{2^{M}} \int_{-\infty}^{+\infty} e^{-\frac{s^{2}}{2}+s \sqrt{\frac{\beta}{M}} \sum x_{j}} d s \\
& \text { setting } y=\sqrt{\frac{\beta}{M}} s \text { we obtain: } \\
= & (2 \pi)^{-1 / 2} Z_{\beta, M}^{-1} \sqrt{\frac{M}{\beta}} \int_{-\infty}^{+\infty} e^{-\frac{M}{2 \beta} y^{2}} \cosh ^{M} y\left(\frac{1}{2^{M} \cosh ^{M} y} \prod_{i=1}^{M} e^{y x_{i}}\right) d y \\
= & (2 \pi)^{-1 / 2} Z_{\beta, M}^{-1} \sqrt{\frac{M}{\beta}} \int_{-\infty}^{+\infty} e^{-M\left(\frac{y^{2}}{2 \beta}-\ln \cosh y\right)} \prod_{i=1}^{M}\left(\frac{e^{y x_{i}}}{\cosh y} P_{0}\left(x_{i}\right)\right) d y
\end{aligned}
$$

a change $t=\tanh y$ of variables gives:

$$
\begin{aligned}
& =(2 \pi)^{-1 / 2} Z_{\beta, M}^{-1} \sqrt{\frac{M}{\beta}} \int_{-1}^{+1} e^{-M F_{\beta}(t) / 2} P_{t}\left(x_{1}, x_{2}, \ldots, x_{M}\right) \frac{1}{1-t^{2}} d t \\
& =Z^{-1} \int_{-1}^{+1} P_{t}^{M}\left(x_{1}, x_{2}, \ldots, x_{M}\right) \frac{e^{-M F_{\beta}(t) / 2}}{1-t^{2}} d t .
\end{aligned}
$$

Above we used that for $|t|<1$ we have $\tanh ^{-1}(t)=\frac{1}{2} \ln \frac{1+t}{1-t}$, $\frac{d t}{d y}=$ $\frac{1}{\cosh ^{2} y}=\frac{\cosh ^{2} y-\sinh ^{2} y}{\cosh ^{2} y}=1-\tanh ^{2} y$, and $\ln \cosh y=-\frac{1}{2} \ln \left(1-\tanh ^{2} y\right)$.

Remark 21 From the above proof an alternative representation of the CurieWeiss probability follows. Defining the measure $Q_{y}=\frac{1}{2 \cosh y}\left(e^{y} \delta_{1}+\right.$ $\left.e^{-y} \delta_{-1}\right)$ and $Q_{y}^{M}$ its $M$-fold product we may write

$$
\mathbb{P}_{\beta, M}\left(x_{1}, x_{2}, \ldots, x_{M}\right)=\widetilde{Z}^{-1} \int_{-\infty}^{+\infty} e^{-M\left(\frac{y^{2}}{2 \beta}+\ln \cosh y\right)} Q_{y}^{M}\left(x_{1}, x_{2}, \ldots, x_{M}\right) d y .
$$

This formula occurs in the physics literature (at least in disguise).

Definition 22 Let $F:(-1,1) \rightarrow \mathbb{R}$ be a measurable function such that $Z=\int_{-1}^{1} \frac{e^{-N F(t) / 2}}{1-t^{2}} d t$ is finite for all $N \in \mathbb{N}$, then the probability measure $\mathbb{P}_{M}^{N F}$ on $\{-1,1\}^{M}$ is defined by

$$
\mathbb{P}_{M}^{N F}\left(x_{1}, x_{2}, \ldots, x_{M}\right)=Z^{-1} \int_{-1}^{+1} P_{t}^{M}\left(x_{1}, x_{2}, \ldots, x_{M}\right) \frac{e^{-N F(t) / 2}}{1-t^{2}} d t
$$

We call a measure of the form $\mathbb{P}_{M}^{N F}$ a generalized Curie-Weiss measure. 
Remark 23 Obviously, $\mathbb{P}_{M}^{N F}$ is a measure of de Finetti type and we have $\mathbb{P}_{\beta, M}=\mathbb{P}_{M}^{M \cdot F_{\beta}}$. Note that $N$ and $M$ may be different in general.

The advantage of the form (25) is that for many cases we can compute the asymptotics of the correlation functions as $N \rightarrow \infty$ using the Laplace method:

Proposition 24 (Laplace method [16]) Suppose $F:(-1,1) \rightarrow \mathbb{R}$ is differentiable and $\phi:(-1,1) \rightarrow \mathbb{R}$ is measurable and for some $a \in(-1,1)$ we have

1. $\inf _{x \in[a, 1]} F(x)=F(a)$ and $\inf _{x \in[b, 1]} F(x)>F(a)$ for all $b \in(a, 1)$.

2. $F^{\prime}$ and $\phi$ are continuous in a neighborhood of a.

3. As $x \searrow$ a we have

$$
\begin{aligned}
F(x) & =F(a)+P(x-a)^{\nu}+\mathcal{O}\left((x-a)^{\nu+1}\right) \\
\phi(x) & =Q(x-a)^{\lambda-1}+\mathcal{O}\left((x-a)^{\lambda}\right)
\end{aligned}
$$

where $\nu, \lambda$ and $P$ are positive constants and $Q$ is a real constant and (26) is differentiable.

4. The integral $I(N)=\int_{a}^{1} e^{-N F(x) / 2} \phi(x) d x$ is finite for all sufficiently large $N$.

Then as $N \rightarrow \infty$

$$
I(N) \approx \frac{Q}{\nu} \Gamma\left(\frac{\lambda}{\nu}\right) P^{-\frac{\lambda}{\nu}}\left(\frac{N}{2}\right)^{-\frac{\lambda}{\nu}} e^{-N F(a) / 2}
$$

where $A(N) \approx B(N)$ means $\lim _{N \rightarrow \infty} \frac{A(N)}{B(N)}=1$ and $\Gamma$ denotes the Gamma function.

Remark 25 This theorem and its proof can be found in [16, Ch. $3 \S 7]$.

We apply the Laplace method to a few interesting cases of $\mathbb{P}_{M}^{N F}$.

Theorem 26 Let $F:(-1,1) \rightarrow \mathbb{R}$ be a smooth even function with $F(t) \rightarrow$ $\infty$ as $t \rightarrow \pm 1$ such that $\int_{-1}^{1} e^{-N F(t) / 2} t^{p} \frac{d t}{1-t^{2}}$ is finite for all $p \geq 0$ and all $N$ big enough and suppose that $F$ has a unique minimum in $[0,1)$ at $t=a$. Then we have for distinct $X_{1}, X_{2}, \ldots, X_{K}, K \leq M$ as $N \rightarrow \infty$ and uniformly in $M$ :

1. If $a=0$ and $F^{\prime \prime}(0)>0$ (i. e. $F$ has a quadratic minimum at 0 ), then for $K$ even:

$$
\mathbb{E}_{M}^{N F}\left(X_{1} X_{2} \ldots X_{K}\right) \approx(k-1) ! ! \frac{1}{\left(\frac{1}{2} F^{\prime \prime}(0)\right)^{K / 2}} \frac{1}{N^{K / 2}}
$$

and for $K$ odd :

$$
\mathbb{E}_{M}^{N F}\left(X_{1} X_{2} \ldots X_{K}\right)=0 .
$$


2. If $a=0$ and $F^{\prime \prime}(0)=0, F^{(4)}(0)>0$ (i. e. $F$ has a quartic minimum at 0 ), then

for $K$ even:

$$
\mathbb{E}_{M}^{N F}\left(X_{1} X_{2} \ldots X_{K}\right) \approx C_{K} \frac{1}{\left(\frac{1}{24} F^{(4)}(0)\right)^{K / 4}} \frac{1}{N^{K / 4}}
$$

and for K odd:

$$
\mathbb{E}_{M}^{N F}\left(X_{1} X_{2} \ldots X_{K}\right)=0
$$

where $C_{K}=\frac{\Gamma\left(\frac{k+1}{4}\right)}{\Gamma\left(\frac{1}{4}\right)} 2^{K / 4}$.

3. If $a>0$ and $F^{\prime \prime}(a)>0$ then

$$
\mathbb{E}_{M}^{N F}\left(X_{1} X_{2} \ldots X_{K}\right) \approx \frac{1}{2}\left(a^{K}+a^{-K}\right) .
$$

Proof. The proof of Theorem 26 relies on the Laplace method (Proposition 24). We concentrate on the proof of case 1, the other cases are proved by the same reasoning.

We set

$$
Z_{K}=\int_{-1}^{+1} e^{-N F(t) / 2} \frac{t^{K}}{1-t^{2}} d t
$$

Then by (25) and Proposition 18 we have $\mathbb{E}_{M}^{N F}\left(X_{1} X_{2} \ldots X_{K}\right)=\frac{Z_{K}}{Z_{0}}$. For $K$ odd we have $Z_{K}=0$ since $\phi(t)=t^{K}$ is odd in this case. For even $K$ we have $Z_{K}=2 \tilde{Z}_{K}$ with $\tilde{Z}_{K}=\int_{0}^{+1} e^{-N F(t) / 2} \frac{t^{K}}{1-t^{2}} d t$. Moreover, $F(t)=F(0)+\frac{t^{2}}{2} F^{\prime \prime}(0)+\mathcal{O}\left(t^{3}\right)$. Applying Proposition 24 both to $\tilde{Z}_{K}$ and to $\tilde{Z}_{0}$ we obtain:

$$
\begin{aligned}
& \tilde{Z}_{K} \approx \Gamma\left(\frac{k+1}{2}\right)\left(\frac{1}{\frac{1}{2} F^{\prime \prime}(0)}\right)^{\frac{K+1}{2}}\left(\frac{2}{N}\right)^{\frac{K+1}{2}} e^{-N F(0)} \\
& \tilde{Z}_{0} \approx \Gamma\left(\frac{1}{2}\right)\left(\frac{1}{\frac{1}{2} F^{\prime \prime}(0)}\right)^{\frac{1}{2}}\left(\frac{2}{N}\right)^{\frac{1}{2}} e^{-N F(0)} .
\end{aligned}
$$

Hence, we get

$$
\begin{aligned}
\mathbb{E}_{M}^{N F}\left(X_{1} X_{2} \ldots X_{K}\right) & =\frac{\tilde{Z}_{K}}{\tilde{Z}_{0}} \\
& \approx \frac{\Gamma\left(\frac{k+1}{2}\right)}{\Gamma\left(\frac{1}{2}\right)} 2^{K / 2}\left(\frac{1}{\frac{1}{2} F^{\prime \prime}(0)}\right)^{\frac{K}{2}}\left(\frac{1}{N}\right)^{\frac{K}{2}}
\end{aligned}
$$

The result (11) then follows from the observation that $\frac{\Gamma\left(\frac{k+1}{2}\right)}{\Gamma\left(\frac{1}{2}\right)} 2^{K / 2}=$ $(K-1)$ !! for even $K$. Case 2 can be handled in a similar way. 
For case 3 we note that $-a$ is also a minimum of the function $F$ since $F$ is even. We devide the integral $\int_{-1}^{1}$ into four parts, namely $\int_{-1}^{-a}+\int_{-a}^{0}+\int_{0}^{a}+\int_{a}^{1}$ and observe that each of these terms has the same asymptotics as $N \rightarrow \infty$.

Remark 27 As a remark to the above proof we notice that under the assumptions in case 1 we have

$$
\left(\int_{-1}^{+1} e^{-\frac{N F(t)}{2}} \frac{d t}{1-t^{2}}\right)^{-1} \int_{-1}^{+1} e^{-\frac{N F(t)}{2}} \frac{|t|}{1-t^{2}} d t \approx \frac{\sqrt{2}}{\sqrt{\pi}} \frac{1}{\sqrt{\frac{1}{2} F^{\prime \prime}(0)}} \frac{1}{\sqrt{N}}
$$

and in case 2 we obtain

$$
\left(\int_{-1}^{+1} e^{-\frac{N F(t)}{2}} \frac{d t}{1-t^{2}}\right)^{-1} \int_{-1}^{+1} e^{-\frac{N F(t)}{2}} \frac{|t|}{1-t^{2}} d t \approx C_{\beta} \frac{1}{N^{1 / 4}} .
$$

Corollary 28 Let $F_{\beta}(t)=\frac{1}{\beta}\left(\frac{1}{2} \ln \frac{1+t}{1-t}\right)^{2}+\ln \left(1-t^{2}\right)$ and let $M(N)$ be a function of $N$ and $K \leq M(N)$ for $N$ large enough and let $X_{1}, X_{2}, \ldots, X_{K}$ be a sequence of distinct random variables. As before we set

$$
\mathbb{E}_{M(N)}^{N F_{\beta}}(\cdot)=Z^{-1} \int_{-1}^{+1} E_{t}^{M(N)}(\cdot) \frac{e^{-\frac{N F(t)}{2}}}{1-t^{2}} d t .
$$

1. For $\beta<1$ we have

$$
\begin{aligned}
\text { for K even: } & \\
\mathbb{E}_{M(N)}^{N F_{\beta}}\left(X_{1} X_{2} \ldots X_{K}\right) & \approx(k-1) ! !\left(\frac{\beta}{1-\beta}\right)^{K / 2} \frac{1}{N^{K / 2}} \\
\text { for } K \text { odd }: & \\
\mathbb{E}_{M(N)}^{N F_{\beta}}\left(X_{1} X_{2} \ldots X_{K}\right) & =0 .
\end{aligned}
$$

2. For $\beta=1$ we have for a constant $c_{K}>0$ :

$$
\begin{array}{lll}
\text { for } K \text { even: } & \mathbb{E}_{M(N)}^{N F_{1}}\left(X_{1} X_{2} \ldots X_{K}\right) \approx c_{K} \frac{1}{N^{K / 4}} \\
\text { for } K \text { odd: } & \mathbb{E}_{M(N)}^{N F_{1}}\left(X_{1} X_{2} \ldots X_{K}\right)=0 .
\end{array}
$$

3. For $\beta>1$ we have

$$
\mathbb{E}_{M(N)}^{N F_{\beta}}\left(X_{1} X_{2} \ldots X_{K}\right) \approx \frac{1}{2}\left(m(\beta)^{K}+(-m(\beta))^{K}\right)
$$

where $m(\beta)>0$ is the unique positive solution of $\tanh (\beta t)=t$. 
Proof. Let us compute the minima of the function $F_{\beta}$. We have:

$$
F_{\beta}^{\prime}(t)=\frac{1}{1-t^{2}}\left(\frac{1}{\beta} \ln \frac{1+t}{1-t}-2 t\right)
$$

hence the possible extrema $m$ of $F_{\beta}$ satisfy:

$$
\frac{1}{2} \ln \frac{1+m}{1-m}=\beta m
$$

or equivalently

$$
\tanh \beta m=m \text {. }
$$

For $\beta<1$ the only solution is $m=0$ and this solution is a quadratic minimum since $F_{\beta}^{\prime \prime}(0)=2 \frac{1-\beta}{\beta}>0$ for $\beta<1$.

For $\beta=1$ the solution $m=0$ is a quartic minimums as $F_{1}^{\prime \prime}(0)=0$ and $F_{1}^{(4)}(0)=4$.

For $\beta>1$ the solution $m=0$ is a maximum of $F_{\beta}$ and there is a positive solution $m$ which is a minimum. The same is true for $-m$.

With this information we can apply Theorem 26.

Now, we discuss random matrix ensembles defined through generalized Curie-Weiss models.

Definition 29 Suppose $\alpha>0$ and $F:(-1,1) \rightarrow \mathbb{R}$ is a smooth even function with $F(t) \rightarrow \infty$ as $t \rightarrow \pm 1$ and such that $\int_{-1}^{1} e^{-N^{\alpha} F(t) / 2} t^{p} \frac{d t}{1-t^{2}}$ is finite for all $p \geq 0$ and all $N$ big enough. Let $\left\{Y_{N}(i, j)\right\}_{1 \leq i, j \leq N}$ be a quadratic scheme of $\mathbb{P}_{N^{2}}^{N^{\alpha}} F_{\text {-distributed random variables, and set } X_{N}}(i, j)=$ $Y_{N}(i, j)$ for $i \leq j$ and $X_{N}(i, j)=Y_{N}(j, i)$ for $i>j$. Then we call the random matrix ensemble $X_{N}(i, j)$ a generalized $\left(\mathbb{P}_{N^{2}}^{N^{\alpha}} F\right)$-Curie-Weiss ensemble.

Remark 30 The full Curie-Weiss ensemble is a $\mathbb{P}_{N^{2}}^{N^{2} F_{\beta}}$-ensemble.

Theorem 31 Suppose the random matrix ensemble $X_{N}(i, j)$ is a generalized $\mathbb{P}_{N^{2}}^{N^{\alpha}} F_{\text {-Curie-Weiss ensemble. }}$

1. If $F$ has a unique quadratic minimum at $a=0$ and $\alpha \geq 1$ then the semicircle law holds for $X_{N}$.

2. If $F$ has a unique quartic minimum at $a=0$ and $\alpha \geq 2$ then the semicircle law holds for $X_{N}$. 


\section{Largest eigenvalue}

At a first glance one might expect that for matrix ensembles with generalized Curie-Weiss distribution the limit density of states measure $\mu$ should depend on $\beta$, even for $\beta \leq 1$. After all, the correlation structure of the ensemble depends strongly on $\beta$ : the behavior of the covariance is given by $\mathbb{E}_{\beta, M}\left(X_{1} X_{2}\right) \approx \frac{\beta}{1-\beta} \frac{1}{M}$. However, the result that the limiting eigenvalue distribution does not depend on $\beta$ (as long as $\beta \leq 1$ ) is connected with the fact that

$$
\frac{1}{N} \mathbb{E}_{\beta, N^{2}}\left(\operatorname{tr}\left(\frac{X_{N}}{N^{1 / 2}}\right)^{2}\right)=1
$$

for Curie-Weiss ensembles independent of $\beta \in \mathbb{R}$. In fact, whenever we have $\mathbb{E}\left(X_{N}(i, j)\right)=0$ and $\mathbb{E}\left(X_{N}(i, j)^{2}\right)=1$ the symmetry of the matrix implies

$$
\frac{1}{N} \mathbb{E}\left(\operatorname{tr}\left(\frac{X_{N}}{N^{1 / 2}}\right)^{2}\right)=\frac{1}{N^{2}} \sum_{i, j} \mathbb{E}\left(X_{N}(i, j) X_{N}(j, i)\right)=1 .
$$

Thus, whenever the limiting measure $\sigma$ exists (and has enough finite moments) it must have second moment $\int t^{2} d \sigma=1$.

In this section we investigate the matrix norm

$$
\left\|A_{N}\right\|=\left\|\frac{X_{N}}{N^{1 / 2}}\right\|=\max _{1 \leq i \leq N}\left|\lambda_{i}\left(A_{N}\right)\right|=\max \left(\left|\lambda_{1}\left(A_{N}\right)\right|,\left|\lambda_{N}\left(A_{N}\right)\right|\right)
$$

for the Curie-Weiss and related ensembles. For the 'classical' Curie-Weiss ensemble $\mathbb{P}_{N^{2}}^{N^{2} F_{\beta}}$ we have:

Proposition 32 There is a constant $C$ such that for all $\beta<1$

$$
\limsup _{N \rightarrow \infty} \mathbb{E}_{N^{2}}^{N^{2} F_{\beta}}\left(\left\|A_{N}\right\|\right) \leq C
$$

Proof. The expectation value of the matrix norm $\left\|A_{N}\right\|$ is given by

$$
\mathbb{E}_{N^{2}}^{N^{2} F_{\beta}}\left(\left\|A_{N}\right\|\right)=Z^{-1} \int_{-1}^{+1} E_{t}^{N^{2}}\left(\left\|A_{N}\right\|\right) \frac{e^{-N^{2} F_{\beta}(t) / 2}}{1-t^{2}} d t .
$$

Using the $N \times N$-matrix

$$
\mathcal{E}_{N}=\left(\begin{array}{cccc}
1 & 1 & \ldots & 1 \\
1 & 1 & \ldots & 1 \\
\vdots & \vdots & & \vdots \\
1 & 1 & \ldots & 1
\end{array}\right)
$$


we estimate

$$
E_{t}^{N^{2}}\left(\left\|A_{N}\right\|\right) \leq E_{t}^{N^{2}}\left(\left\|A_{N}-\frac{t}{\sqrt{N}} \mathcal{E}_{N}\right\|\right)+\frac{|t|}{\sqrt{N}}\left\|\mathcal{E}_{N}\right\| .
$$

The matrix $D_{N}=A_{N}-\frac{t}{\sqrt{N}} \mathcal{E}_{N}$ has random entries $D_{N}(i, j)$ which are independent and have mean zero with respect to the probability measure $P_{t}^{N^{2}}$. Thus we may apply [15] (after splitting $D_{N}$ into a lower and uper triangular part) and conclude that $E_{t}^{N^{2}}\left(\left\|D_{N}\right\|\right) \leq C$ for a constant $C<\infty$.

The matrix $\mathcal{G}_{N}=\frac{1}{N} \mathcal{E}_{N}$ represents the orthogonal projection onto the one dimensional subspace generated by the vector $\eta_{N}=\frac{1}{\sqrt{N}}(1,1, \ldots, 1)$. Thus $\left\|\mathcal{G}_{N}\right\|=1$ and $\left\|\mathcal{E}_{N}\right\|=N$. From Remark 27 we learn that

$$
Z^{-1} \int_{-1}^{+1}|t| \frac{e^{-N^{2} F_{\beta}(t) / 2}}{1-t^{2}} d t \approx C_{1} \frac{1}{N}
$$

Thus

$$
\lim \sup _{N \rightarrow \infty} \mathbb{E}_{N^{2}}^{N^{2} F_{\beta}}\left(\left\|A_{N}\right\|\right) \leq \limsup _{N \rightarrow \infty}\left(C+C_{1} \frac{1}{\sqrt{N}}\right)=C .
$$

The borderline case of generalized Curie-Weiss ensembles for Theorem 5 is the measure $\mathbb{E}_{N^{2}}^{N F_{\beta}}$. For this case the expected value of the matrix norm does depend on $\beta$ and goes to infinity as $\beta<1$ tends to 1 .

Proposition 33 For $\beta<1$ we have for positive constants $C_{1}, C_{2}$

$$
\begin{aligned}
\left(\frac{\beta}{1-\beta}\right)^{\frac{1}{2}} C_{1}-C_{2} & \leq \liminf _{N \rightarrow \infty} \mathbb{E}_{N^{2}}^{N F_{\beta}}\left(\left\|A_{N}\right\|\right) \\
\leq \limsup _{N \rightarrow \infty} \mathbb{E}_{N^{2}}^{N F_{\beta}}\left(\left\|A_{N}\right\|\right) & \leq\left(\frac{\beta}{1-\beta}\right)^{\frac{1}{2}} C_{1}+C_{2} .
\end{aligned}
$$

Proof. The argument is close to the proof of the previous Proposition 32 We prove the lower bound, the upper bound is similar.

With the notation of the previous proof we have

$$
\begin{aligned}
E_{t}^{N^{2}}\left(\left\|A_{N}\right\|\right) & \geq \frac{|t|}{\sqrt{N}}\left\|\mathcal{E}_{N}\right\|-E_{t}^{N^{2}}\left(\left\|A_{N}-\frac{t}{\sqrt{N}} \mathcal{E}_{N}\right\|\right) \\
& \geq|t| \sqrt{N}-C_{2}
\end{aligned}
$$

using again the result of [15] and $\left\|\mathcal{E}_{N}\right\|=N$.

Thus

$$
\mathbb{E}_{N^{2}}^{N F_{\beta}}\left(\left\|A_{N}\right\|\right) \geq Z^{-1} \sqrt{N} \int_{-1}^{+1}|t| \frac{e^{-N F_{\beta}(t) / 2}}{1-t^{2}} d t-C_{2} .
$$


From Remark 27 we learn that

$$
Z^{-1} \int_{-1}^{+1}|t| \frac{e^{-N F_{\beta}(t) / 2}}{1-t^{2}} d t \approx C_{1}\left(\frac{\beta}{1-\beta}\right)^{1 / 2} \frac{1}{\sqrt{N}}
$$

hence

$$
\liminf _{N \rightarrow \infty} \mathbb{E}_{N^{2}}^{N F_{\beta}}\left(\left\|A_{N}\right\|\right) \geq\left(\frac{\beta}{1-\beta}\right)^{\frac{1}{2}} C_{1}-C_{2}
$$

We turn to the case of strong correlations, in particular, we consider the full Curie-Weiss ensemble with inverse temperature $\beta>1$. It is easy to see that for a full Curie-Weiss ensemble $X_{N}(i, j)$ with inverse temperature $\beta>1$ the 'averaged traces'

$$
\frac{1}{N} \mathbb{E}_{N^{2}}^{N^{2} F_{\beta}}\left(\operatorname{tr}\left(\frac{X_{N}}{N^{1 / 2}}\right)^{k}\right)
$$

cannot converge for $k$ large enough, in fact we have:

Proposition 34 Consider the random matrix $B^{(\alpha)}=\frac{X_{N}}{N^{\alpha}}$, with $X_{N}$ symmetric and distributed according to the the full Curie-Weiss ensemble with $\beta>1$. Then for $\alpha<1$ and $k$ large enough and even we have

$$
\frac{1}{N} \mathbb{E}_{N^{2}}^{N^{2} F_{\beta}}\left(\operatorname{tr}\left(\frac{X_{N}}{N^{\alpha}}\right)^{k}\right) \rightarrow \infty \quad \text { as } N \rightarrow \infty
$$

and for all $k \geq 1$

$$
\frac{1}{N} \mathbb{E}_{N^{2}}^{N^{2} F_{\beta}}\left(\operatorname{tr}\left(\frac{X_{N}}{N}\right)^{k}\right) \rightarrow 0 \quad \text { as } N \rightarrow \infty .
$$

Proof. We compute using (29)

$$
\begin{aligned}
& \frac{1}{N} \mathbb{E}_{N^{2}}^{N^{2} F_{\beta}}\left(\operatorname{tr}\left(\frac{X_{N}}{N^{\alpha}}\right)^{k}\right) \\
& =\frac{1}{N^{1+k \alpha}} \sum_{i_{1}, i_{2}, \ldots i_{k}} \mathbb{E}_{N^{2}}^{N^{2} F_{\beta}}\left(X_{N}\left(i_{1}, i_{2}\right) X_{N}\left(i_{2}, i_{3}\right) \ldots X_{N}\left(i_{k}, i_{1}\right)\right) \\
& \geq \frac{1}{N^{1+k \alpha}} \sum_{\rho\left(i_{1}, i_{2}, \ldots i_{k}\right)=k} \mathbb{E}_{N^{2}}^{N^{2} F_{\beta}}\left(X_{N}\left(i_{1}, i_{2}\right) X_{N}\left(i_{2}, i_{3}\right) \ldots X_{N}\left(i_{k}, i_{1}\right)\right) \\
& \geq \frac{1}{N^{1+k \alpha}} C N^{k} m(\beta)^{k} \rightarrow \infty \quad \text { for } k \text { large },
\end{aligned}
$$


where again $m(\beta)$ denotes the unique positive solution of $\tanh (\beta t)=t$. We used above, that for all correlations

$$
\mathbb{E}_{N^{2}}^{N^{2} F_{\beta}}\left(X_{N}\left(i_{1}, i_{2}\right) X_{N}\left(i_{2}, i_{3}\right) \ldots X_{N}\left(i_{k}, i_{1}\right)\right) \geq 0 .
$$

The second assertion of the Proposition follows from

$$
\begin{aligned}
& \frac{1}{N} \mathbb{E}_{N^{2}}^{N^{2} F_{\beta}}\left(\operatorname{tr}\left(\frac{X_{N}}{N}\right)^{k}\right) \\
& =\frac{1}{N^{1+k}} \sum_{i_{1}, i_{2}, \ldots i_{k}} \mathbb{E}_{N^{2}}^{N^{2} F_{\beta}}\left(X_{N}\left(i_{1}, i_{2}\right) X_{N}\left(i_{2}, i_{3}\right) \ldots X_{N}\left(i_{k}, i_{1}\right)\right) \\
& \leq \frac{1}{N^{1+k}} N^{k} \rightarrow 0 .
\end{aligned}
$$

Above we used that there are at most $N^{k}$ summand in the above sum.

From Proposition 34 we conclude that the eigenvalue distribution function of $\frac{X_{N}}{N}$ converges to the Dirac measure $\delta_{0}$, while for $\frac{X_{N}}{N^{\alpha}}(\alpha<1)$ at least the moments do not converge. For $\beta>1$ the dependence ('interaction') between the $X_{N}(i, j)$ is so strong that a macroscoping portion of the random variables is aligned, i.e. either most of the $X_{N}(i, j)$ are equal to +1 or most of the $X_{N}(i, j)$ are are equal to -1 and there are about $m(\beta) N^{2}$ more aligned spins than others. Moreover, for large $\beta$, the matrix $\frac{X_{N}}{N}$ should be close to the matrix

$$
\mathcal{G}_{N}=\frac{1}{N} \mathcal{E}_{N}
$$

or to $-\mathcal{G}_{N}$. This intuition is supported by the following observation.

Proposition 35 Let $B_{N}=\frac{X_{N}}{N}$ with $X_{N}$ distributed according to $\mathbb{P}_{N^{2}}^{N^{2} F_{\beta}}$, then

1. For $\beta<1$ we have $\left\|B_{N}\right\| \rightarrow 0$ in probability.

2. For $\beta>1$ we have $\left\|B_{N}\right\| \rightarrow m(\beta)$ in probability.

Proof. Part 1 follows from $\left\|B_{N}\right\|=\frac{1}{\sqrt{N}}\left\|A_{N}\right\|$ and from the estimate $\sup _{N} \mathbb{E}_{N^{2}}^{N^{2} F_{\beta}}\left(\left\|A_{N}\right\|\right)<\infty$ by Proposition 33 .

To prove 2 we start with an estimate from below. We set $\eta_{N}=\frac{1}{\sqrt{N}}(1, \ldots, 1)$ 
and use the short hand notation $\mathbb{E}$ instead of $\mathbb{E}_{N^{2}}^{N^{2} F_{\beta}}$.

$$
\begin{aligned}
\mathbb{E}\left(\left\|B_{N}\right\|^{2}\right) & \geq \mathbb{E}\left(\left\|B_{N} \eta_{N}\right\|^{2}\right) \\
& =\frac{1}{N^{3}} \sum_{i=1}^{N} \mathbb{E}\left|\sum_{j=1}^{N} X_{N}(i, j)\right|^{2} \\
& =\frac{1}{N^{2}} \sum_{j, k=1}^{N} \mathbb{E}\left(X_{N}(1, j) X_{N}(1, k)\right) \\
& =\frac{1}{N^{2}}\left(1+N(N-1) \mathbb{E}\left(X_{N}(1,1) X_{N}(1,2)\right) \rightarrow m(\beta)^{2}\right.
\end{aligned}
$$

since $\mathbb{E}\left(X_{N}(1,1) X_{N}(1,2)\right) \rightarrow m(\beta)^{2}$ for $\beta>1$ by Proposition 28 , It follows that

$$
\liminf _{N \rightarrow \infty} \mathbb{E}\left(\left\|B_{N} \eta_{N}\right\|^{2 k}\right) \geq \liminf _{N \rightarrow \infty} \mathbb{E}\left(\left\|B_{N}\right\|^{2}\right)^{k} \geq m(\beta)^{2 k} .
$$

We prove the converse inequality. For $k>1$ we have

$$
\begin{aligned}
& \mathbb{E}\left(\left\|B_{N}\right\|^{2 k}\right) \leq \mathbb{E}\left(\operatorname{tr} B_{N}^{2 k}\right) \\
= & \frac{1}{N^{2 k}} \mathbb{E}\left(\sum_{i_{1}, i_{2}, \ldots, i_{2 k}} X_{N}\left(i_{1}, i_{2}\right) X_{N}\left(i_{2}, i_{3}\right) \ldots X_{N}\left(i_{2 k}, i_{1}\right)\right) .
\end{aligned}
$$

Let $\rho=\rho\left(i_{1}, i_{2}, \ldots, i_{2 k}\right)$ denote the number of different indices among the $i_{j}$, i. e. $\rho\left(i_{1}, i_{2}, \ldots, i_{2 k}\right)=\#\left\{i_{1}, i_{2}, \ldots, i_{2 k}\right\}$, then

$$
\frac{1}{N^{2 k}} \mathbb{E}\left(\sum_{\rho\left(i_{1}, i_{2}, \ldots, i_{2 k}\right)<2 k} X_{N}\left(i_{1}, i_{2}\right) X_{N}\left(i_{2}, i_{3}\right) \ldots X_{N}\left(i_{2 k}, i_{1}\right)\right) \rightarrow 0
$$

while

$$
\frac{1}{N^{2 k}} \mathbb{E}\left(\sum_{\rho\left(i_{1}, i_{2}, \ldots, i_{2 k}\right)=2 k} X_{N}\left(i_{1}, i_{2}\right) X_{N}\left(i_{2}, i_{3}\right) \ldots X_{N}\left(i_{2 k}, i_{1}\right)\right) \rightarrow m(\beta)^{2 k}
$$

by Proposition 28

We also have

$$
\mathbb{E}\left(\left\|B_{N}\right\|^{2}\right) \leq \mathbb{E}\left(\left\|B_{N}\right\|^{4}\right)^{1 / 2}
$$

Thus we have proved that

$$
\mathbb{E}\left(\left\|B_{N}\right\|^{2 k}\right) \rightarrow m(\beta)^{2 k}
$$

for all $k \in \mathbb{N}$. It follows that $\left\|B_{N}\right\|^{2}$ converges in distribution to $\delta_{m(\beta)^{2}}$, hence $\left\|B_{N}\right\|$ converges in distribution to $\delta_{m(\beta)}$, therefore it converges in probability to $m(\beta)$. 


\section{References}

[1] D. Aldous: Exchangeability and related topics, pp. 1-198 in: Lecture Notes in Mathematics 117, Springer (1985).

[2] G. Anderson, A. Guionnet, O. Zeitouni: An introduction to random matrices, Cambridge University Press (2010).

[3] L. Arnold: On Wigner's semicircle law for the eigenvalues of random matrices, Z. Wahrsch. Verw. Gebiete 19, 191-198 (1971).

[4] Z. Bai, J. Silverstein: Spectral analysis of large dimensional random matrices, Springer (2010).

[5] J. Baik, G. Ben Arous, S. Péché: Phase transition of the largest eigenvalue for nonnull complex sample covariance matrices, Ann. Prob. 33, 1643-1697 (2005).

[6] W. Bryc, A. Dembo, T. Jiang: Spectral measure of large random Hankel, Markov and Toeplitz matrices, Ann. Prob. 34, 1-38 (2006).

[7] S. Chatterjee: A generalization of the Lindeberg principle, Ann. Probab. 34, 2061-2076 (2006).

[8] R. Ellis: Entropy, Large Deviations, and Statistical Mechanics, Springer (1985).

[9] B. de Finetti: Funzione caratteristica di un fenomeno aleatorio, Atti della R. Academia Nazionale dei Lincei, Serie 6. Memorie, Classe di Scienze Fisiche, Mathematice e Naturale, 4, 251-299 (1931).

[10] O. Friesen, M. Löwe: The Semicircle Law for Matrices with Independent Diagonals, J. Theoret. Probab. 26, 1084-1096 (2013).

[11] O. Friesen, M. Löwe: A phase transition for the limiting spectral density of random matrices, Electron. J. Probab. 18, 1-17 (2013).

[12] F. Götze, A. Naumov, A. Tikhomirov: Semicircle law for a class of random matrices with dependent entries, Preprint arXiv:1211.0389v2.

[13] F. Götze, A. Tikhomirov: Limit theorems for spectra of random matrices with martingale structure, Theory Probab. Appl. 51, 42-64 (2007).

[14] W. Kirsch: A review of the moment method, in preparation.

[15] R. Latała: Some estimates of norms of random matrices, Proc. Amer. Math. Soc. 133, 1273-1282 (2005).

[16] F. Olver: Asymptotics and special functions, Academic Press (1974).

[17] L. Pastur: Spectra of random selfadjoint operators, Russian Math. Surveys 28, 1-67 (1973).

[18] L. Pastur, M. Sherbina: Eigenvalue distribution of large random matrices, Mathematical Surveys and Monographs 171, AMS (2011). 
[19] R. Stanley: Enumerative Combinatorics, Vol. 2, Cambridge University Press (1999).

[20] J. Schenker, H. Schulz-Baldes: Semicircle law and freeness for random matrices with symmetries or correlations, Mathematical Research Letters 12, 531-542 (2005)

[21] T. Tao: Topics in random matrix theory, AMS (2012).

[22] C. Thompson: Mathematical Statistical Mechanics, Princeton University Press (1979).

[23] E. Wigner: On the distribution of the roots of certain symmetric matrices, Ann. Math. 67, 325-328 (1958).

Winfried Hochstättler

Werner Kirsch

Simone Warzel winfried.hochstaettler@fernuni-hagen.de werner.kirschefernuni-hagen. de

warzel@ma.tum.de 